\title{
Continuing Research During a Crisis
}

\author{
Kurt Kroenke, $M D^{1,2}$ (0), Matthew J. Bair, MD ${ }^{1,2,3}$, and Greg A. Sachs, $M D^{1,2}$ \\ 'Division of General Internal Medicine and Geriatrics, Department of Medicine, Indiana University School of Medicine, Indianapolis, USA; \\ ${ }^{2}$ Regenstrief Institute, Inc., Room 221, 1101 W. 10th Street, Indianapolis, IN, USA; ${ }^{3}$ VA HSR\&D Center for Health Information and Communication, \\ Roudebush VAMC, Indianapolis, USA.
}

J Gen Intern Med 36(4):1086-8

DOI: $10.1007 / \mathrm{s} 11606-021-06636-5$

(c) Society of General Internal Medicine 2021

$\mathrm{R}$ esearch stands alongside many of the global activities that have been constrained because of the COVID-19 pandemic. Physical distancing policies to mitigate viral spread have banned or restricted in-person contacts for hundreds of thousands of clinical trials as well as other types of human subjects' research, not to mention the temporary shutdown of many laboratory studies. The suspension of scientific investigations that are in progress can invalidate or dramatically influence the quality and interpretation of study results, a ramification magnified in proportion to the moratorium duration. Few people question the necessity of restricting human contacts as much as possible during the outbreak of a novel infectious agent to safeguard human health and to preserve critical health care resources. Now nearly a year into the pandemic, research procedures are only one among many institutional and societal policies requiring regular re-evaluation based upon viral surges that ebb and flow. Even when COVID-19 eventually abates, lessons learned from the current mitigation efforts will hopefully inform strategies regarding research during future regional, national, or global crises.

McDermott and Newman have articulated strategies for maintaining the integrity of clinical trials during the mandatory pandemic-related bans on in-person contacts. ${ }^{1}$ Their pragmatic suggestions include (a) informing study participants of study adjustments that may temporarily affect the study assessments or intervention; (b) modifying evaluations or treatments, when possible, to a method that may be administered remotely by telephone or web-based technologies; (c) for study procedures that cannot be conducted remotely, resume assessments and interventions after the in-person ban is lifted; (d) accounting for and reporting the interrupted subgroup of affected participants in the methods and analysis sections of the manuscript; (e) maintaining or even increasing study activities that do not require in-person contact, such as

Received April 10, 2020

Accepted January 20, 2021

Published online February 8, 2021

determining study eligibility as well as enrolling and consenting participants. Others have highlighted similar as well as additional pandemic-related accommodations. ${ }^{2,3}$

Complementing these highly useful recommendations, we provide some additional suggestions in Table 1. These apply to all types of human subjects' research necessitating inperson or virtual contacts, including clinical trials and epidemiological studies as well as community-engaged, health systems, and translational research. The two major clinical research activities most affected by restrictions on in-person contacts are participant assessments and intervention delivery. These activities are affected differentially by where along the study timeline a participant is situated, i.e., whether proximal to the baseline assessment or an intervention that has not yet begun vs. follow-up assessments and ongoing interventions. Whereas challenges are clearly greatest with the latter group, potential strategies for dealing with these different circumstances are proffered. Pandemic-related constraints on basic science research have been qualitatively different but nonetheless substantial. Although the number of unique human contacts may be fewer for bench than for clinical studies, it is typically not feasible to conduct laboratory experiments remotely using telephone or other virtual technology.

Besides the primary purpose of maintaining the integrity of research, there are secondary benefits to sustaining studies until the pandemic wanes and face-to-face engagement resumes. First, study participants currently isolated from their usual interactions at work, at school, and in the community may value the human contact with members of the research team. Second, research staff remain employed, a non-trivial issue when many businesses and jobs are being jeopardized by the pandemic. Anything that counters the economic fall-out, even to a modest degree, is consequential. Third, keeping study participants involved allows them to contribute to something meaningful and important while their usual daily activities, like jobs, may be curtailed. Fourth, clinician-scientists who are able to keep their research active may experience a temporary and welcome respite from the pressures of pandemic-related clinical activities. Fifth, continuing research may help preserve a sense of normalcy for investigators, staff, and study participants. Sixth, enormous amounts of monies have been committed to ongoing studies by the federal government and other sponsors, and honoring these contractual 
Table 1 Strategies for Continuing a Clinical Research Study When In-Person Contacts Are Restricted

\section{Study component \\ Baseline assessment}

Follow-up assessment

Intervention-subject has not yet started

Intervention-subject has started

\section{Potential strategy}

1. Convert if possible to virtual (remote) assessment by audio (if only patient-reported outcomes are required) or video (if patient observation or performance is required) 2. If study cannot convert to remote until in-person contacts are permitted 1. Same as strategy \#1 for baseline assessment

2. If study cannot convert to remote assessment and has assessments at multiple time points, drop one (or more) of the assessments for these participants. Repeated measures or imputation strategies can deal with missing assessments.

3. If near the end of the in-person ban period, delay the assessment if possible to do so within a reasonable time window

1. Delay intervention until in-person ban has been lifted (and decide if study needs to re-do eligibility interview at that time)

1. Convert if possible to a virtual (remote) intervention delivery

2 . If study cannot convert to remote delivery, suspend for several months until the in-person contact ban is lifted. Then pick up where study left off. For example, if study delivered sessions

1-3 of a 6-session intervention, finish sessions 4-6 after in-person ban is

lifted

3. If study cannot pick up where it left off, consider restarting the intervention after in-person ban is lifted (a downside is a subgroup of participants will get a "double dose" for repeated sessions).

1. Try to avoid dropping enrolled subjects if at all possible

2. Clear major study changes with IRB and study sponsor

3. Enroll a larger number of participants if possible to compensate

for the pandemic-related subgroup should the latter affect the results 4. Use secondary analytic strategies (e.g., analyzing study results with and without pandemic-related participant subgroup; adjusting for pandemic subassessment, delay baseline assessment group as a covariate; etc.)

obligations to the extent possible, while still prioritizing control of the pandemic, benefits not only science but also the study participants and research staff.

It may be helpful to reflect on a different, historical crisis to create some emotional space while at the same time examining the impact on both science and individual scientists. During World War II, some scientists were drafted while others had to decide whether to enlist for service or continue their research during the war. A survey was sent to 122 academic zoologists to examine their attitudes regarding research during the war. ${ }^{4}$ Of 41 respondents, only 6 were in favor of discontinuing research, whereas 27 favored continuing their current research,
5 for continuing it in their spare time, and 3 for redirecting research to war-related problems.

A related albeit not identical decisional dilemma confronts researchers during the current pandemic. Some clinicianscientists are putting research studies on hold not only due to barriers imposed by physical distancing but also because of increased clinical duties necessitated by the pandemic. Senior clinician-scientists either no longer involved in patient care or at greater risk if exposed to the virus are wondering how they can assist the pandemic efforts in ways other than front-line clinical care. ${ }^{5,6}$ Both clinical and basic scientists whose principal activities are not focused on COVID-19 have debated whether they should re-direct (pivot) their skills to pandemicrelated research or assist in research temporarily set aside by their clinician-scientist colleagues whose patient care responsibilities have increased. While wrestling with these decisions, the varying rather than uniform attitudes among scientists during previous crises such as World War II signify that there is unlikely to be one right answer.

Major events such as contagion, war, and natural disasters will inevitably disrupt research to a greater or less degree. The impact may be local (circumscribed disasters), regional or continental (wars or more widespread disasters), and with the current pandemic even global. The disruptive impact will vary with not only the geographic extent but also the type of event. ${ }^{7}$ For example, research challenges in the aftermath of Hurricane Katrina included locating displaced participants, establishing alternative clinical sites and laboratory facilities, and securing specimens and data storage. ${ }^{7}$

Nonetheless, infectious diseases, wars, and disasters will share some common detrimental impacts on research. These include diversion of funds, people, and other resources to more urgent priorities; physical or geographic obstacles to continuing scientific studies; decisional dilemmas for many individuals regarding where and how to devote their time and skills; and negative feelings that can temporarily dampen energy or enthusiasm for research, such as worry, fear, exhaustion, burnout, and even existential concerns. We also may have to be nimbler and more flexible in how research is approved, funded, and conducted during times of crisis. ${ }^{8}$ Finally, it is important that the preferences of research participants already enrolled in studies be considered in weighing the risks and benefits of continued participation. ${ }^{9}$ What we do and learn during COVID-19 and its aftermath will not only help sustain research during the pandemic but in future crises as well. It is our hope that research and unanticipated societal disruptions may co-exist rather than be mutually exclusive.

Corresponding Author: Kurt Kroenke, MD; Regenstrief Institute, Inc., Room 221, 1101 W. 10th Street, Indianapolis, IN 46202, USA (e-mail: kkroenke@regenstrief.org). 


\section{REFERENCES}

1. McDermott MM, Newman AB. Preserving clinical trial integrity during the coronavirus pandemic. JAMA. 2020;323:2135-2136.

2. Fleming TR, Labriola $\mathbf{D}$, Wittes J. Conducting clinical research during the COVID-19 pandemic: protecting scientific integrity. JAMA 2020;324:33-34.

3. Lumeng JC, Chavous TM, Lok AS, Sen S, Wigginton NS, Cunningham RM. Opinion: A risk-benefit framework for human research during the COVID-19 pandemic. Proc Natl Acad Sci 2020 Nov 10;117(45):27749-53.

4. Stern C. Peacetime research in wartime. Science 1944;99:278-280.

5. Buerhaus PI, Auerbach DI, Staiger DO. Older clinicians and the surge in novel coronavirus disease 2019 (COVID-19). JAMA 2020;323:1777-1778.

6. Reuben DB. Sideline guilt. JAMA Intern Med 2020 180(9):1150-1.
7. McDuffie R, Summerson J, Reilly P, Blackwell C, Goff D, Kimel AR, Crago L, Fonseca V. The Action to Control Cardiovascular Risk in Diabetes (ACCORD) Trial and Hurricane Katrina: Lessons for managing clinical trials during and after a natural disaster. Contemp Clin Trials 2008; 29(5):756-61.

8. Angus DC. Optimizing the trade-off between learning and doing in a pandemic. JAMA 2020;323:1895-1896.

9. Padala PR, Jendro AM, Padala KP. Conducting clinical research during the COVID-19 pandemic: investigator and participant perspectives. JMIR Public Health Surveill 2020;6(2):e18887. doi: https://doi.org/10.2196/ 18887

Publisher's Note Springer Nature remains neutral with regard to jurisdictional claims in published maps and institutional affiliations. 